\title{
CORONA DURING THE TOTAL SOLAR ECLIPSE ON MARCH 20, 2015, AND 24 CYCLE DEVELOPMENT
}

\author{
S.A. Yazev ${ }^{1,2}$, A.V. Mordvinov ${ }^{2}$, A.A. Dvorkina-Samarskaya ${ }^{1}$ \\ ${ }^{1}$ Irkutsk State University, Irkutsk, Russia,syazev@gmail.com \\ ${ }^{2}$ Institute of Solar-Terrestrial Physics SB RAS, Irkutsk, Russia, avm@iszj.irk.ru
}

We analyzed the structure of coronal features, using data on the March 20, 2015 total solar eclipse. The Ludendorff index characterizing the flattening of the corona is 0.09. The solar corona structure in the northern and southern hemispheres corresponds to the maximum and post-maximum phases of solar activity, respectively. The asynchronous development of magnetic activity in the Sun's northern and southern hemispheres caused a substantial asymmetry of coronal features observed at the reversal of polar magnetic fields in the current cycle. The polar ray structures in the southern hemisphere are associated with the polar coronal hole, while in the northern hemisphere a polar hole has not been formed yet. We examine the relation between large-scale magnetic fields and location of high coronal structures.

Keywords: Total solar eclipse, asymmetry, solar corona.

\section{INTRODUCTION}

During total solar eclipses, the solar corona structure visualizes the Sun's large-scale magnetic field [Nesmyanovich, 1965; Kuchmi et al., 2011]. The analysis of corona images provided new information about cyclic variations in the Sun's global magnetic field [Klepikov, Filippov, 2006; Judge et al., 2010], and revealed secular variations in the solar corona structure [Tlatov, 2010; Mordvinov et al., 2011]. To study the solar corona structure is still important for heliophysics, especially in the context of the unusual development of solar activity in the current cycle.

The Russian astronomer A.P. Hansky was probably one of the first to establish in 1897 a relation between a shape of the solar corona during an eclipse and a phase of an 11-year solar cycle [Hansky, 1897, VorontsovVelyaminov, 1956; Perel, 1951]. Analyzing solar eclipse images, including those he took himself during the expedition to Novaya Zemlya in 1896, A.P. Hansky noted that during solar activity maximum the corona encircled the Sun with a homogeneous aureole, and during solar minimum it extended along the solar equator. He suggested that coronal rays were spatially linked with prominences. The analysis of corona images captured during 12 solar eclipses allowed A.P. Hansky to distinguish three main morphological types of the solar corona: maximum, intermediate, and minimum. 
A more detailed classification of types of the solar corona based on images stored in the late XIX - early XX was set forth by the Kiev astronomer A.T. Nesmyanovich [Nesmyanovich, 1965]. His approach considered the index of solar corona flattening as a function of sunspot cycle phase $F$ on the date of an eclipse:

$$
F=\frac{T-T_{\text {min }}}{\left|T_{\max }-T_{\min }\right|},
$$

where $T$ is the moment of a total eclipse, $T_{\max }$ and $T_{\min }$ are respective maximum and subsequent minimum of a solar cycle (during the decay phase) or maximum and preceding minimum of the cycle (during the growth phase) expressed in months. Values of $F$ in such an approach appear to be positive when solar activity goes up and negative when it goes down.

We present this classification in Figure 1 and Table 1 [Nesmyanovich, 1965]. Notice that the classification is rather rough, but overall it represents basic periodicities of variations in the observed shape of the corona in the plane of the sky during a solar cycle. The cause of these variations is generally thought to be associated with inclinations of the heliospheric current sheet (HCS) from the helioequatorial plane such that in the general case they are related also to the phase of a cycle [Gulyaev, 1992; Makarov et al., 1996; Gulyaev, 1997; Koomen et al., 1998; Peshcherov et al., 2006]. At the same time, some inclinations of HCS from the equator were registered; they were anomalous for this phase. They produced shapes of the solar corona inconsistent with Nesmyanovich's classification [Gulyaev, 1992; Gulyaev, 1997; Peshcherov et al., 2006]. Nevertheless, the authors believe that the said classification in a first approximation can be used to assess the situation.

Yet another total solar eclipse occurred on March 20, 2015. An expedition organized by Irkutsk State University observed the solar corona during the eclipse from two points of the island Western Spitsbergen: from the slope of the mountain Pyramiden at an altitude of $400 \mathrm{~m}$ and from the gulf coast.

This paper reports results of the analysis of new solar corona images captured during the postmaximum phase of the sunspot cycle. The purpose of the paper is to analyze the solar corona structure seen on March 20, 2015, determine its type from Nesmyanovich's classification, and compare it with the structure of large-scale magnetic fields on the Sun. We think that this study contributes to the heliophysical database on the development of solar cycle 24 .

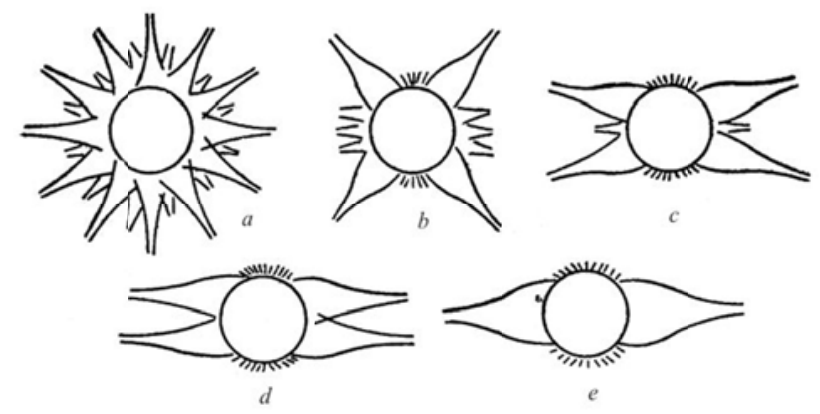

Figure 1. Types of the corona during an eclipse according to A.T. Nesmyanovich [Nesmyanovich, 1965]. Maximum (a), pre-maximum or post-maximum $(b)$, pre-minimum or post-minimum $(c)$, minimum $(d)$, ideally minimum (e) 
Table 1. Types of the solar corona according to A.T. Nesmyanovich

\begin{tabular}{|l|l|l|l|l|}
\hline 1 & \multicolumn{1}{|c|}{$\begin{array}{c}\text { Type of the solar } \\
\text { corona }\end{array}$} & \multicolumn{1}{c|}{ Polar ray systems } & \multicolumn{1}{c|}{ Coronal rays } & Phase \\
\hline 11 & maximum & unobserved & radial, at all latitudes & $|F|>0.85$ \\
\hline 22 & $\begin{array}{l}\text { pre-maximum or } \\
\text { post-maximum }\end{array}$ & $\begin{array}{l}\text { observed at least } \\
\text { in one hemisphere }\end{array}$ & $\begin{array}{l}\text { radial or slightly inclined, } \\
\text { at high latitudes }\end{array}$ & $0.5<|F|<0.85$ \\
\hline 33 & $\begin{array}{l}\text { pre-minimum or } \\
\text { post-minimum }\end{array}$ & $\begin{array}{l}\text { well developed } \\
\text { in both hemispheres }\end{array}$ & $\begin{array}{l}\text { strongly inclined toward } \\
\text { the equatorial plane }\end{array}$ & $0.15<|F|<0.5$ \\
\hline 44 & minimum & $\begin{array}{l}\text { highly developed } \\
\text { in both hemispheres }\end{array}$ & $\begin{array}{l}\text { parallel to the equatorial } \\
\text { plane }\end{array}$ & $|F|<0.15$ \\
\hline 55 & ideally minimum & $\begin{array}{l}\text { highly developed } \\
\text { in both hemispheres }\end{array}$ & $\begin{array}{l}\text { located along the } \\
\text { equatorial plane }\end{array}$ & $|F|<0.05$ \\
\hline
\end{tabular}

\section{PREDICTION OF THE SOLAR CORONA MORPHOLOGY} DURING THE MARCH 20, 2015 ECLIPSE

According to Nesmyanovich's approach, phenomenology (type) of the corona depends on the phase of a cycle $F$. Current solar cycle 24 began in January 2009 and reached its maximum in April 2014, after 64 months (5.3 years). Such duration of the growth phase $T_{\mathrm{r}}$ has precedents characteristic of the period of the late XIX - early XX. For example, $T_{\mathrm{r}}$ was 4.0 years in solar cycle 15, 4.5 years in solar cycle $13,4.8$ years in solar cycle 16, 5.0 years in solar cycle 12, and 5.3 years in solar cycle 14. Thus, the current cycle is similar in some of its characteristics to cycles 12-16. This provides grounds for assuming that sunspot cycle 24 can develop under the scenario of one of the cycles (compared cycles). This allows us to give an estimate of its expected duration.

Yazev [Yazev, 2012] noted that variations in average monthly Wolf numbers $W$ during the current cycle corresponded almost exactly to their variations in sunspot cycle 16 (1923-1933). The author presented a plot analogous to Figure 2 with the last point corresponding to August 2012; the correlation coefficients between the curves were 0.96. However, from October 2012 (the 46th month from the beginning of the cycle), the curves lost their synchronism (they were almost in antiphase, Figure 3). A year later, in October 2013, there was the second powerful burst of activity ensuring the principal maximum of the cycle. Thus, the scenario of solar cycle 16 occurred again. The difference is that solar cycle 16 displayed a burst of activity between two main maxima - current cycle 24 did not have this burst. In general, we should note a high degree of similarity between the curves describing development of both the cycles, including identical "age" and amplitudes (Figure 2). This circumstance allows us to use for comparison plots drawn from average monthly data, not smoothed curves as is normally done.

The curve that describes solar cycle 24 is sufficiently similar to those for solar cycles 12 and 14 with respect to average monthly Wolf numbers $W$ (Figure 3). While the similarity between cycles 12 and 24 during the growth phase turned out to be worse than for solar cycle 16, approximately in the 50th month after the beginning of the cycle the degree of similarity became sufficiently high (Figure $3, a$ ). The same is true for solar cycle 14 (Figure 3, b). 


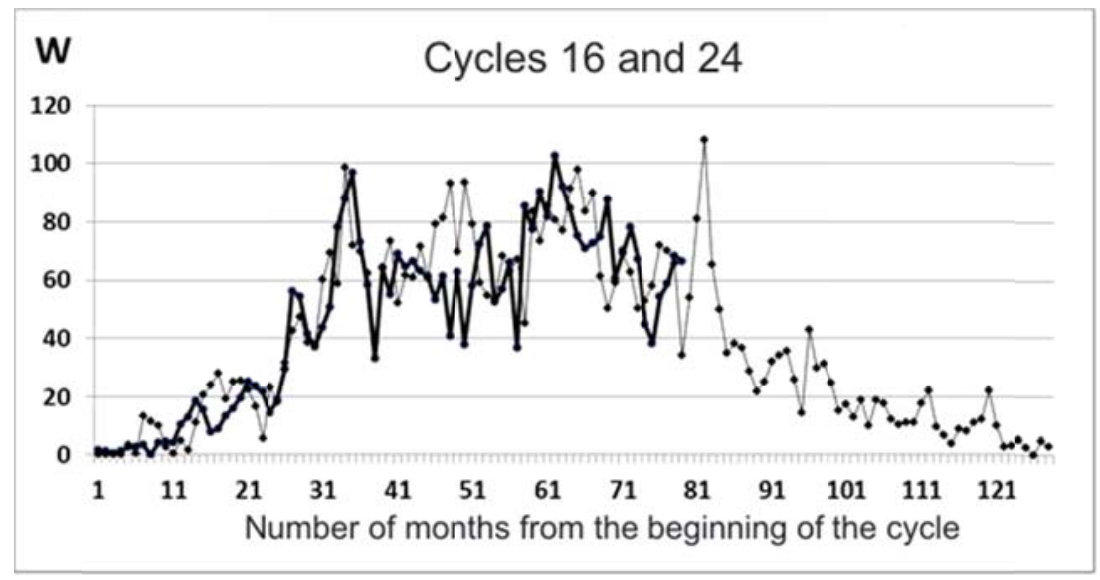

Figure 2. Development of solar cycles 24 (thick line) and 16 (thin line) expressed in average monthly Wolf numbers. The last point on the $\mathrm{X}$-axis for solar cycle 24 refers to July 2015
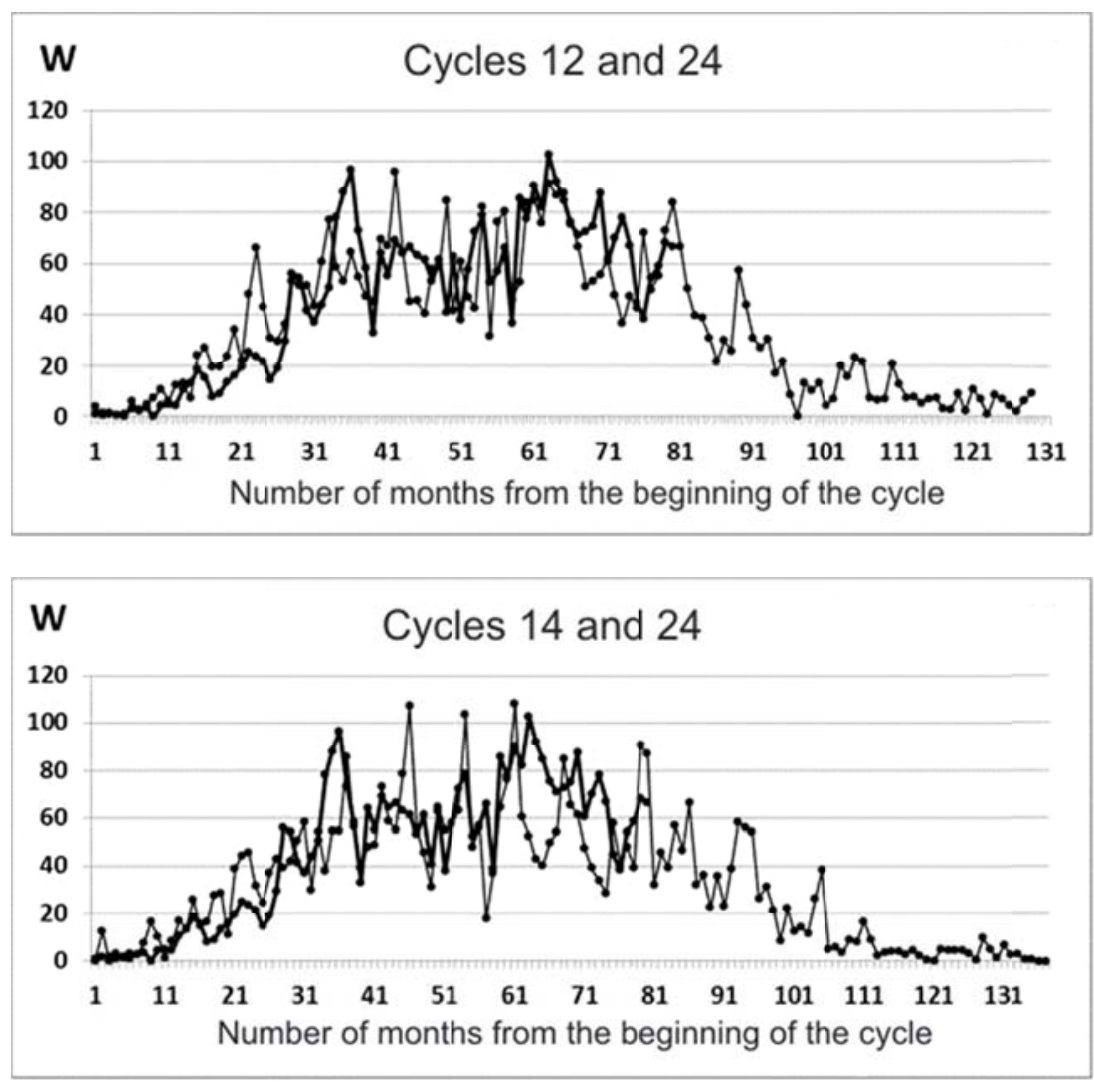

Figure 3. Evolution of solar cycles 24 (thick line, $a, b$ ), 12 (thin line, $a$ ), and 14 (thin line, $b$ ) expressed in average monthly Wolf numbers. The last point on the X-axis for solar cycle 24 refers to July 2015

Supposing that parameters of the current solar cycle are close to those of low cycles 12,14 , and 16, we can calculate the phase $F$ of the total eclipse on March 20, 2015 for the maximum phase of solar cycle 24 from the duration of the compared cycles.

Assuming that the moment of the eclipse $T=67$ months after the beginning of the cycle, $T_{\max }$ of the cycle was registered in the 64th month (Figure 1), then according to Formula (1) we can calculate $F$ values corresponding to the moment of the eclipse from the expected duration of the current cycle under the scenario of the three compared cycles. We give the results in Table 2. 
Table 2. Phase of cycle on the date of eclipse depending on cycle scenario

\begin{tabular}{|c|c|c|c|c|}
\hline Cycle scenario & $\begin{array}{c}\text { Duration } \\
\text { of the growth phase } T_{\mathrm{r}} \text {, years }\end{array}$ & $\begin{array}{c}\text { Duration of cycle } \\
\text { years/months }\end{array}$ & $F$ & $\begin{array}{c}\text { Expected } \\
\text { type of the } \\
\text { solar corona }\end{array}$ \\
\hline 16 & 4.8 & $10.2 / 122.4$ & 0.95 & 1 \\
\hline 14 & 5.3 & $11.9 / 142.8$ & 0.96 & 1 \\
\hline 12 & 5.0 & $10.7 / 128.4$ & 0.95 & 1 \\
\hline
\end{tabular}

Under any of the three compared scenarios (types of cycles 16, 14, or 12), $F$ for the March 20, 2015 eclipse appeared to be $0.95-0.96$. According to Table 1 , this phase corresponds to the maximum type of the solar corona (Figure 1,a).

\section{CORONA MORPHOLOGY ON MARCH 20, 2015 AS INFERRED FROM OBSERVATIONS MADE DURING THE EXPEDITION}

Figure 4 illustrates a solar corona image synthesized from a series of photographs with different exposure time. We constructed this image by normalizing the local brightness to radius-averaged values. To reduce noise and intensify low-contrast coronal features, we utilized two-dimensional wavelet filtration of normalized image. Such processing allowed us to find the fine structure of the solar corona scarcely distinguishable in the composite image constructed from original photographs (Figure 5).

Pishkalo [Pishkalo, 2011] has summarized 170 Ludendorff indices $\varepsilon$ characterizing flattening of isophots of the solar corona $r=2 R_{\mathrm{s}}$, where $R_{\mathrm{S}}$ is the solar radius. This index numerically describes the shape of the solar corona in a quasielliptic approximation. Isophots have been drawn by many authors throughout the century, using drawings and photographs of the solar corona for 60 eclipses observed from 1851 to 2010. The author [Pishkalo, 2011] confirmed the conclusion, deduced by a number of authors, that $\varepsilon$ systematically varies with the phase of cycle from zero (during maximum solar activity) to 0.4 (during minimum solar activity).

For the series of corona images captured on March 20, 2015 we obtained a number of isophots with the maximum equatorial radius equal to $2 R_{\mathrm{s}}$ (Figure 5). We calculated the Ludendorff index $\varepsilon=0.09$ from an outer isophot, using a method described in [Pishkalo, 2011]. According to the summary [Pishkalo, 2011], analogous values were registered during the eclipses on December 4, 2002 with $F=0.71$ (type 2 of the solar corona), June 8, 1937 with $F=0.95$ (type 1 of the solar corona), and February 16, 1980 with $F=0.97$ (type 1 of the solar corona). We can assert that $\varepsilon=0.09$ is typical of the maximum phase of the solar cycle.

Nevertheless, a more detailed analysis of the solar corona structure from the eclipse pictures has shown that the conclusion on the maximum type of the solar corona drawn from the Ludendorff index can be considered only partly true (Figures 4,5 ).

From the solar corona images, we have selected at least nine clearly defined high coronal structures, including six ones for the northern hemisphere (Figure 4). Their basic characteristics are given in Table 3. 


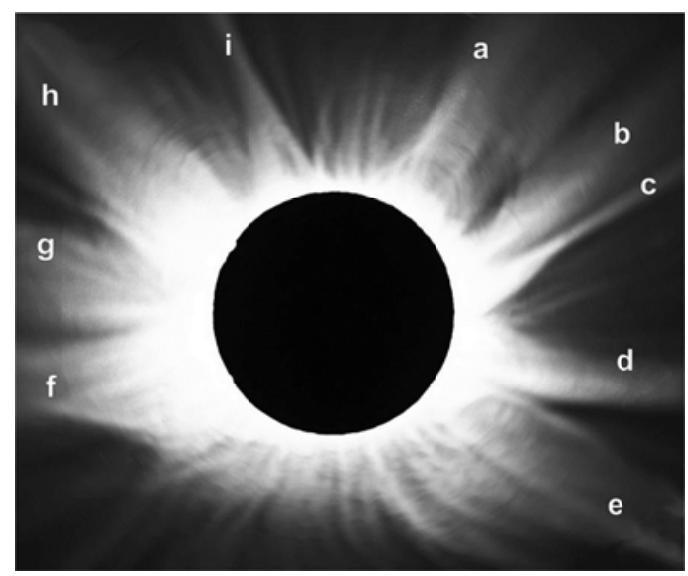

Figure 4. The solar corona during the March 20, 2015 eclipse: a composite image based on a series of photographs taken by M.V. Chekulaev. Letters a-i indicate the longest coronal rays

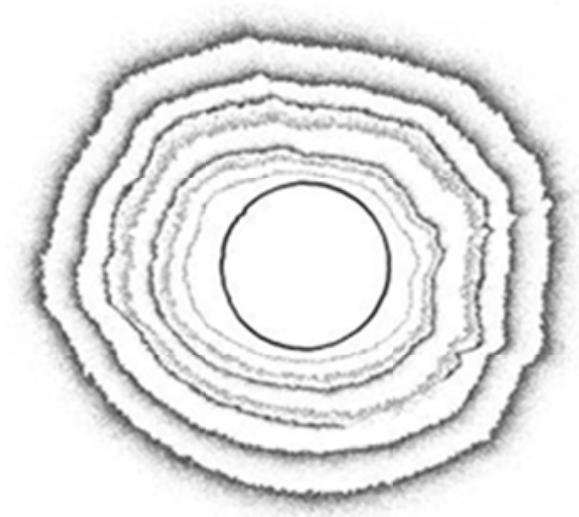

Figure 5. The solar corona on March 20, 2015. We drew the isophots, using the series of pictures taken by M.G. Gavrilov, V.V. Ryabenko, and M.V. Chekulaev

Table 3. Properties of coronal structures during the March 20, 2015 eclipse

\begin{tabular}{|l|c|c|c|l|}
\hline Structure & $\begin{array}{c}\text { Position } \\
\text { on the limb }\end{array}$ & $\begin{array}{c}\text { Latitude } \\
\text { of the base } \\
\text { on the limb }\end{array}$ & $\begin{array}{c}\text { Height } \\
\text { above the limb } \\
R_{\mathrm{s}}\end{array}$ & \multicolumn{1}{|c|}{ Structural properties } \\
\hline a & $\mathrm{NW}$ & $50^{\circ} \mathrm{N}$ & 1.0 & Radial ray \\
\hline $\mathrm{b}$ & $\mathrm{NW}$ & $17^{\circ} \mathrm{N}$ & 1.6 & Radial ray \\
\hline $\mathrm{c}$ & $\mathrm{NW}$ & $8^{\circ} \mathrm{N}$ & 2.1 & Radial ray \\
\hline $\mathrm{g}$ & $\mathrm{NE}$ & $27^{\circ} \mathrm{N}$ & 1.6 & Radial ray \\
\hline $\mathrm{h}$ & $\mathrm{NE}$ & $42^{\circ} \mathrm{N}$ & 1.5 & $\begin{array}{l}\text { coronal streamer, wide radial ray, } \\
\text { bright prominence at the base }\end{array}$ \\
\hline $\mathrm{i}$ & $\mathrm{NE}$ & $82^{\circ} \mathrm{N}$ & 1.3 & High-latitude radial ray \\
\hline $\mathrm{d}$ & $\mathrm{SW}$ & $25^{\circ} \mathrm{S}$ & 1.9 & $\begin{array}{l}\text { coronal ray extended along a position angle, } \\
\text { inclination toward the equatorial plane }\end{array}$ \\
\hline $\mathrm{e}$ & $\mathrm{SW}$ & $57^{\circ} \mathrm{S}$ & 1.5 & $\begin{array}{l}\text { coronal ray, inclination toward the } \\
\text { equatorial plane }\end{array}$ \\
\hline $\mathrm{f}$ & $\mathrm{SE}$ & $20^{\circ} \mathrm{S}$ & 1.8 & $\begin{array}{l}\text { coronal ray extended along a position angle, } \\
\text { inclination toward the equatorial plane }\end{array}$ \\
\hline
\end{tabular}

Structures (coronal rays) are lettered; their position on the limb (quadrants) is shown in the second column. The third column gives latitudes of the center of the base of a coronal ray on the limb. For the rays $\mathbf{d}, \mathbf{f}$ extended along the position angle, we can roughly estimate this latitude. The maximum height of rays above the limb expressed in solar radii (the fourth column) was determined from original solar 
corona images taken by V.V. Ryabenko and M.V. Gavrilov with maximum exposure ( $0.1 \mathrm{~s})$. The real height of rays is much greater; this refers to the brightest areas found in original (unprocessed) images. The fifth column presents additional characteristics of coronal structures.

For the northern hemisphere, we can conclude that the solar corona in this hemisphere corresponds to type 1 of the A.T. Nesmyanovich's classification (Table 1). Specifically, the coronal rays a, b, c, g, h, and $\mathbf{i}$ are radial, located at all latitudes, including high ones (the southern boundary of the $\mathbf{i}$ ray is on the east limb; the northern one, on the west limb; the projection of the ray onto the plane of the sky is nearly over the pole). The northern hemisphere exhibits no polar ray structures.

Unlike the northern hemisphere, it is difficult to suggest that the corona in the southern hemisphere belongs to the maximum type. The wide diffused coronal ray $\mathbf{d}$ rather less bright in original pictures inclines considerably from the radial direction toward the equatorial plane. The relatively narrow ray e also inclines toward the equatorial plane. The east limb of the southern hemisphere revealed the diffused ray $\mathbf{f}$ extended along a position angle $\left(20^{\circ} \mathrm{N}\right)$. It also inclined noticeably from the radial direction toward the equatorial plane. There was a prominence at the base of this ray.

There are no high coronal rays at higher latitudes of the southern hemisphere. The pictures show polar ray structures quasiradial near the pole and inclining toward the equatorial plane (toward the east on the east limb and toward the west on the west one) as the latitude lowers.

According to Table 1, all the above properties are typical for the type 3 solar corona (post-maximum corona) featuring relatively low-latitude coronal rays inclining toward the equatorial plane and the polar ray structure at the pole. The March 20, 2015 solar corona seems to be "composed of halves": the northern hemisphere corresponds to type 1 ; the Southern one, to type 2.

Thus, the phenomenological characteristics of the solar corona during the March 20, 2015 eclipse probably belong formally to none of the types according to A.T. Nesmyanovich's classification. Obviously, this is associated with the considerable north-south asymmetry of the solar activity development appearing at the end of cycle 23 and observed during solar cycle 24 [Yazev, 2015].

In particular, one of the manifestations of this asymmetry is as follows. Already by June 2014 at the Sun's south pole, there appeared an extensive polar coronal hole asymmetric about the heliographic pole. It presumably facilitated the development of polar ray structures tracing bases of lines in the open field extending from the polar region high up to the corona.

In months that followed, this coronal hole expanded and became ever more contrast. In the northern hemisphere during the eclipse, there were high-latitude (to $40^{\circ}$ and higher) prominences on the limb and filaments on the disc (Figure 6). High prominences (diffuse filaments) separate regions with different polarity of the radial component of magnetic field. This means that a large-scale unipolar magnetic region capable of generating a new coronal hole has not been formed yet. 


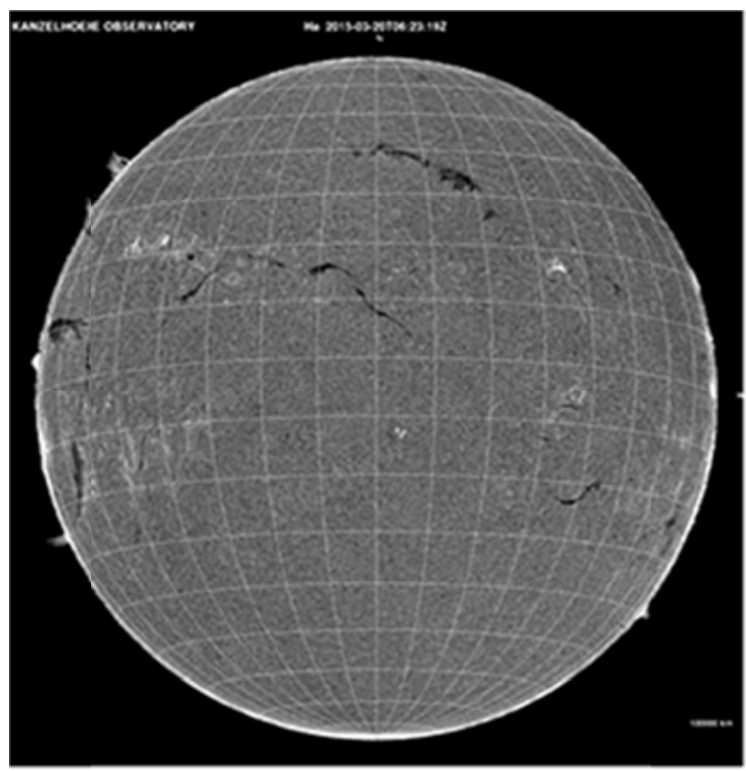

Figure 6. The Sun during the eclipse in the Ha line, Kanzelhoehe observatory

We can assume that the low sunspot activity in the northern hemisphere in 2013-2014 caused the transfer of residual tail fields of active regions to high latitudes to become less intensive. This, in turn, led to the delay in the formation of the coronal hole and respective magnetic field configuration in the polar zone. As a result, during the eclipse there were no polar ray structures, but there existed high-latitude coronal rays.

\section{FINE STRUCTURE OF THE SOLAR CORONA AND THE SUN'S MAGNETIC FIELD}

The Sun's large-scale magnetic field influences physical processes in its atmosphere, determining largely the structure and shape of the corona [Kuchmi et al., 2011]. Figure 7 pictures the fine structure of the corona during the March 20, 2015 total solar eclipse; the composite corona image was taken by M. Druckmüller using an original method [Druckmüller, 2013]. On the solar disc, closed contour lines depict boundaries separating polarities of large-scale magnetic fields as deduced from VSM/SOLIS data. Along these boundaries are chromospheric filaments; their shape is shown by black thick line segments. Signs $«+»$ and «-» mark regions occupied with magnetic fields of respective polarities.

Closed contour lines with dashes show boundaries of coronal holes $(\mathrm{CH})$. The observed $\mathrm{CH}$ are located in unipolar magnetic regions (UMR); their characteristic inclination toward the helioequator indicates that they were formed by meridional flows and differential rotation of the Sun [Wang et al., 2007; Eselevich et al., 1999]. The polar CH at the south pole is situated in a vast UMR of negative polarity that was formed by the meridional transfer of residual magnetic fields after decay of long-lived activity complexes observed during maximum magnetic activity in the southern hemisphere [Mordvinov, Yazev, 2013; Mordvinov, Yazev, 2014; Mordvinov et al., 2015]. In the polar zone of the northern hemisphere, there is a small $\mathrm{CH}$ located inside an UMR of positive polarity. Structures of these highlatitude features also depend on the differential rotation and meridional flows. 


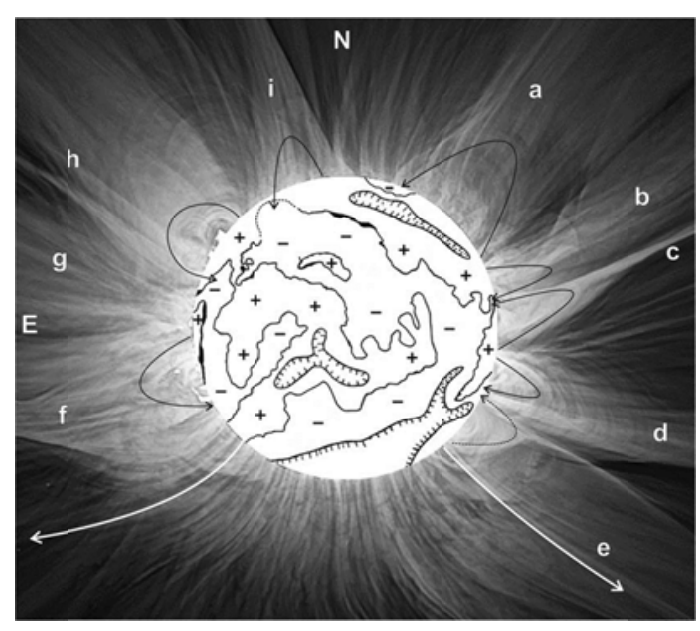

Figure 7. Fine structure of the solar corona, large-scale magnetic fields, and coronal holes on the Sun on March 20, 2015. We present the composite corona image with consent of the author (M. Druckmüller)

In general, as mentioned above, the solar corona has a shape characteristic of the period of maximum activity. There is also a north-south asymmetry of coronal structures. The long rays and streamers (helmets) form the corona on the east, north, and west sides, while nearby the south pole there exists a system of polar plumes. Thus, in the northern hemisphere, the solar corona has a structure typical for maximum activity; and in the southern hemisphere, its shape corresponds to the phase of transition from maximum to minimum activity.

Solar corona images taken during eclipses allow us to directly compare individual coronal features with magnetic fields on the solar surface.

It is known that coronal ray structures visualize magnetic field lines in the Sun's atmosphere. The most extended ray structures are observed over coronal arches with bases located in a region of opposite polarity magnetic fields. Figure 7 presents a scheme of magnetic field lines (indicated by black arrows) with respect to the sign of photospheric fields and properties of the fine structure of coronal features. Such a scheme conforms to current notions of typical magnetic field configurations existing in streamer belts and chains [Wang et al., 2007; Eselevich et al., 1999].

Nearby the north pole, there was a streamer at the base of the coronal ray a. One of the photospheric bases of the streamer is located in the negative polarity region with its most part being out of the limb. This situation illustrates the complex structure of the magnetic field in the polar zone of the northern hemisphere and explains why a stable coronal hole has not emerged at the north pole yet.

The coronal rays $\mathbf{b}, \mathbf{c}$ and $\mathbf{d}, \mathbf{e}$ are related to the streamers with double magnetic arches at their bases. Such structures are generally associated with streamer chains (pseudostreamers); their magnetic structure is shown by arrows in the figure. The dashed arrow indicates an arch whose apparent bases are projected onto the regions of negative polarity magnetic field. Still, 24 hours before the eclipse near the south base of the streamer, there existed a positive polarity region. During the eclipse, it was hidden behind the limb. Thus, coronal rays are related to the chain of streamers with opposite edges of positive polarity. 


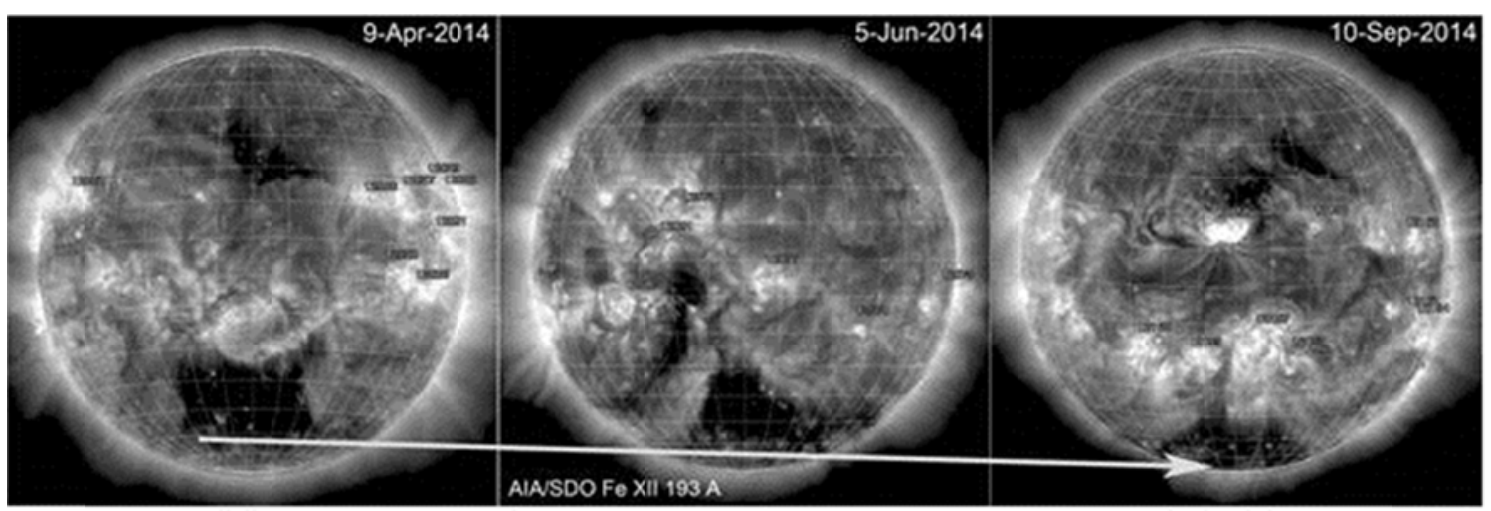

Figure 8 . Formation of a polar coronal hole at the south pole of the Sun

Nearby the Sun's south pole, there were polar plumes; their edges are shown by white arrows. Photospheric bases of the polar plumes lie in a vast UMR of positive polarity with a stable $\mathrm{CH}$; its evolution is illustrated in Figure 8.

\section{CONCLUSION}

The comprehensive analysis of the data acquired during the March 20, 2015 total solar eclipse allowed us to study the solar corona structure with respect to photospheric magnetic fields. We have established that the corona structure in the northern hemisphere corresponds to the period of maximum activity; in the southern hemisphere, to the post-maximum period.

Observations of the solar corona during the eclipse immediately after the solar polarity reversal have revealed peculiarities of large-scale magnetic fields that influence the formation of polar coronal holes. We have advanced arguments in favor of the assumption that the coronal features observable during the eclipse were caused by the asynchronous development of magnetic activity in the Sun's northern and southern hemispheres. The considerable north-south asymmetry of solar activity in the current cycle produced differences in the structure of magnetic fields in the Sun's polar regions. These peculiarities clearly manifested themselves in the asymmetry of polar $\mathrm{CH}$ : at the south pole, a stable $\mathrm{CH}$ emerged at the end of 2014, whereas nearby the north pole there exist only short-lived primordial $\mathrm{CH}$.

The coronal rays $\mathbf{f}, \mathbf{h}$, and $\mathbf{i}$ are associated with the streamers having bases located in opposite polarity regions. The magnetic structure of these formations is schematized by arrows. Hence, the streamer belt on the east side of the Sun is situated over a large-scale neutral line; along this line there exist prominences seen on the limb.

The study was partially supported by the project of the Ministry of Education and Science of the Russian Federation No. 3.615.2014/K. We express gratitude to participants of the expedition under scientific supervision of S.A. Yazev - M.G. Gavrilov, A.L. Manannikov, M.A. Merkulov, V.V. Ryabenko, D.V. Semenov, E.D. Skaredneva, and M.V. Chekulaev for acquisition of observational data during the eclipse. 


\section{REFERENCES}

Druckmüller M. A noise adaptive fuzzy equalization method for processing solar extreme ultraviolet images. Astrophys. J. Suppl. 2013, vol. 207, article id. 25, 5 p. DOI: 10.1088/0067-0049/207/2/25.

Eselevich V.G., Fainshtein V.G., Rudenko G.V. Study of the structure of streamer belts and chains in the solar corona. Solar Phys. 1999, vol. 188, pp. 277-297.

Gulyaev R.A. Outer solar corona as an optical manifestation of heliospheric current sheet. Uspekhi Fizicheskikh Nauk [Physics-Uspekhi [Adv. in Phys. Sci.]. 1992, vol. 162, iss. 12, pp. 155-159. (In Russian).

Gulyaev R.A. Solar cycle variation on the solar corona shape: A new outlook. Astron. Astrophys. Trans. 1997, vol. 13, no. 2, pp. 137-144.

Hansky. Die totale Sonnenfinsterniss am 8 August 1896. Bull. Acad. Imper. Sci. St._Petersbourg, 1897, vol. 6, pp. 251-270.

Judge P.G., Burkepile J., de Toma G., Druckmüller M. Historical eclipses and the recent solar minimum corona. ASP Conf. Ser. 2010, vol. 428, pp. 171-176.

Klepikov D.V., Filippov B.P. Behavior of the polar field magnetic focuses in solar cycle from SOHO/EIT. Izvestiya RAN [Bull. Russian Academy of Sciences: Physics]. 2006, vol. 70, pp. 1436-1438. (In Russian).

Koomen M.J., Howard R.A., Michels D.J. The shape of the outer corona during cycle 21. Solar Phys. 1998, vol. 180, iss. 1/2, pp. 247-263. DOI: 10.1023/A:1005039609265.

Kuchmi S.L., Merzlyakov V.L., Molodensky M.M. On 3D-structure of the solar corona. Astronomicheskii Zhurnal [Astron. J.]. 2001, vol. 78, no. 10, pp. 953-960. (In Russian).

Makarov V.I., Tlatov A.G., Fatianov M.P. Three-dimensional modeling of the corona in different phases of solar magnetic cycle. Period: 1870-1991. Izvestiya Vysshikh Uchebnykh Zavedenii. Radioelektronika [Radioelectronics and Communications Systems]. 1996, vol. 39, no. 10, pp. 1268-1274. (In Russian).

Mordvinov A.V., Yazev S.A. Complexes of activity decay and formation of coronal holes on the Sun. Astronomicheskii Zhurnal [Astron. J.]. 2013, vol. 90, no. 6, pp. 491-500. (In Russian).

Mordvinov A.V., Yazev S.A., Rykova E.G., Dvorkina-Samarskaya A.A. Long-term changes in geometry of the Sun's polar magnetic field from observation of total solar eclipses. Solnechno-Zemnaya Fizika [Solar-Terrestrial Physics]. 2011, iss. 18, pp. 69. (In Russian).

Mordvinov A.V., Yazev S.A. Reversals of the Sun's polar magnetic fields in relation to activity complexes and coronal holes. Solar Phys. 2014, vol. 289, pp. 1971-1981.

Mordvinov A.V., Grigoryev V.M., Erofeev D.V. Evolution of sunspot activity and inversion of the Sun's polar magnetic field in the current cycle. Adv. Space Res. 2015, vol. 55, iss. 11, pp. 2739-2743. DOI: 10.1016/j.asr.2015.02.013.

Perel Yu.G. Famous Russian Astronomers. Moscow, State publishing house of technical-theoretical literature, 1951, pp. 194-211. (In Russian).

Peshcherov V.S., Yazev S.A., Ozhogina O.A., et al. Solar corona observations during March 29, 2006 solar eclipse. Selected Problems in Astronomy: Materials of theoretical and practical conference "Sky and Earth" (Irkutsk, November 2123, 2006). Irkutsk: Irkutsk State University Publ., 2006, pp. 151-156. (In Russian).

Pishkalo M. Flattering Index of the solar corona and the solar cycle. Solar Phys. 2011, vol. 270, iss. 1, pp. 347-363. DOI: $10.1007 / \mathrm{s} 11207-011-9749-\mathrm{y}$.

Tlatov A.G. The centenary variations in the solar corona shape in accordance with the observations during the minimal activity epoch. Astron. Astrophys. 2010, vol. 522, id. A27, 5 pp. DOI: 10.1051/0004-6361/201014082.

Vsekhsvyatsky S.K., Nikolsky G.M., Ivanchuk V.I., Nesmyanovich A.T., Ponomarev E.A., Rubo G.A., Cherednichenko V.I. Solnechnaya Korona i Korpuskulyarnoe Izluchenie v Mezhplanetnom Prostranstve [Solar Corona and Corpuscular Radiation in the Interplanetary Space]. Kiev, Kiev University Publ., 1965. 216 c. (In Russian).

Vorontsov-Vel'yaminov B.A. Ocherki Istorii Astronomii v Rossii [Essays in the History of Astronomy in Russia]. Moscow: State publishing house of technical-theoretical literature, 1956, pp. 327-329. (In Russian). 
Wang, Y.-M., Sheeley, N.R.J., Rich, N.B. Coronal pseudo-streamers. Astrophys. J. 2007, vol. 658, iss. 2 , pp. 1340-1348. DOI: 10.1086/511416/

Yazev S.A. Catalogue of complexes of activity on the Sun at the growth phase of Schwabe-Wolf 24 cycle. Izvestiya Irkutskogo Gosudarstvennogo Universiteta. Seria "Nauki o Zemle” [Bulletin of Irkutsk State University. Earth Sciences Ser.]. 2012, vol. 5, no. 2, pp. 295-303. (In Russian).

Yazev S.A. Complexes of activity on the Sun in 24 cycle of solar activity. Astronomicheskii Zhurnal [Astron. J.]. 2015, vol. 92, no. 3, pp. 260-269. (In Russian). 